\title{
Superhydrophobicity of micro-structured surfaces on Zirconia by nanosecond pulsed laser
}

Yukui Cai, Wenlong Chang, Xichun Luo*, Yi Qin

Centre for Precision Manufacturing, DMEM, University of Strathclyde, Glasgow, UK

\begin{abstract}
This paper presents a systematic approach to improve the hydrophobicity of microstructured surfaces. It includes a contact angle prediction model for microstructures obtained by nanosecond pulsed laser. Combining with the theoretical constraints for stable Cassie-Baxter state this approach can be used to optimize microstructures dimensions for maximising surface hydrophobicity. Laser machining experiments were conducted to evaluate the prediction model. It shows that the proposed systematic approach can accurately predict the contact angle and obtain microstructures dimensions for maximising surface hydrophobicity. The results also indicate that the contact angle increases with the decrease of pitch of the microstructures. Superhydrophobicity with maximum contact angle of $155.7^{\circ}$ is obtained, for the first time, on a micro structured surface (P030) of Zirconia with a pitch of $30 \mu \mathrm{m}$ machined under a laser power at $8 \mathrm{~W}$.
\end{abstract}

Keywords: Superhydrophobic surface, microstructures, Zirconia, laser surface structuring 


\section{Introduction}

Zirconia $\left(\mathrm{ZrO}_{2}\right)$ is widely used in dentistry for orthodontic brackets, implant abutments, crowns and bridges, etc. due to its high strength, toughness, low wear, high resistance to corrosion and good esthetical effects [1,2]. At present, dentures made of zirconia are usually fabricated by using tungsten carbide milling cutters with polishing as the final machining process in order to achieve a good surface finish. Scotti found that the polished surface accumulated fewer bacteria than a glazed surface possibly because fine surface roughness on dental materials reduces the bacterial cell more than on irregular surfaces [3]. Some researchers have verified that surface roughness causes enhanced bacterial adhesion by supplying harbour within the surface irregularities $[4,5]$.

However, recent investigation found that nano/micro scale surface texturing alters surface characteristics, causing them to be hydrophobic and moreover antibacterial [6]. Hydrophobic and super-hydrophobic surfaces have received significant attention because of their anti-icing, drag reduction, corrosion protection and anti-bacteria properties [7, 8]. Yilbas found that textured surface composed of micro/nano poles on silicon materials would improve the surface hydrophobicity significantly [9]. Thus, surface structure modification is an effective way to obtain superhydrophobic surface. Different machining processes, such as micro milling $[10,11]$, electrochemical deposition/chemical vapour deposition $[12,13]$, lithography [14] or laser machining $[15,16]$ have been used to prepare such microstructures. However, most of these processes have limited feasibility of preparing microstructures on zirconia. The attainable dimension of the microstructures by using micro milling machining is restricted by the available smallest diameter of milling cutters. Thus, micro milling process is difficult to obtain structures at micron level. Moreover, rapid tool wear or tool breakage is another problem when micro milling Zirconia which is a kind of difficult-to-machine material due to its high hardness and strength. Electrochemical deposition/ chemical vapour deposition has 
low stability and repeatability in machining microstructures. Lithography is an expensive and low-efficiency process due to the high cost in the fabrication of micro structured template, while nanosecond laser machining process has been found to be a very promising costeffective method for surface texturing due to its high-efficiency and contactless characteristics [17-20].

Rajab examined a stainless steel surface processed by using a laser machining and found that the stainless steel surface with the oval shaped and rounded topped surface features was the best surfaces with less number of bacteria retention [21]. Tong's research showed that the distribution of staphylococcus aureus on surfaces possessing high contact angles would become scattered. The phenomenon became even more apparent for superhydrophobic surfaces in which the contact angle is larger than $150^{\circ}$ [22]. Kwon integrated a series of machining processes including laser machining and electrodeposition processes to manufacture micro structures on stainless steel and found that the maximum contact angle that could be achieved was $153^{\circ}$ [23]. Yang [24] created superhydrophobic surface on Inconel 718 with maximum contact angle of $156^{\circ}$ by using a nanosecond laser. In these studies, achievement of superhydrophobicity heavily relies on the right choice of microstructures, either nature-inspired non-periodic microstructures which are usually difficult to machine or brain-stormed periodic microstructures. Due to the lack of clear understanding of relationship between dimensions of microstructures with surface hydrophobicity costly and time consuming "trial and error approach" has to be resorted to find out optimized dimension in these studies.

Zirconia is naturally a kind hydrophilic material. Currently the achieved maximum contact angle is only $132^{\circ}[25,26]$ for laser structured Zirconia surfaces due to the lack of a systematic approach to design the microstructures to achieve superhydrophobic surfaces (i.e. contact angle larger than $\left.150^{\circ}\right)$. 
This study will focus on the establishment of a prediction model for laser machined microstructured surface and an optimization approach to design micro structure dimensions to maximize its hydrophobicity. It will enable generate superhydrophobic microstructures on Zirconia by the high-efficiency and low-cost nanosecond laser machining process.

\section{Modelling of Cassie-Baxter state for micro pillar arrays}

Two typical models, Wenzel and Cassie-Baxter models, have been developed to describe the behavior of a droplet on rough surfaces $[27,28]$ in the study of hydrophobicity. According to the Wenzel model, the droplet penetrates the structures. Alternatively, the droplet is not able to wet the microstructure spaces for the Cassie-Baxter model. Thus, the droplet should have a stable Cassie-Baxter state on the specimen surface in order to obtain the Zirconia specimen possessing good superhydrophobicity. In this section, the condition of water droplet with stable and robust Cassie-Baxter state will be investigated.

Due to the Gaussian distribution of the laser beam the cross section of the laser machined micro groove will be also like a Gaussian curve as shown in Fig. 1 (a). The 3D geometrical models of laser machined Gaussian pillar arrays are shown in Fig. 1 (b). In Fig. 1(a), $a$ is the depth of the Gaussian groove, $b$ is the width of the micro pillar, and $6 c$ is the width of micro groove. $h$ is the sag in height of droplet between pillars. $\theta$ is the intrinsic contact angle of zirconia. We assuume that the sag of the water droplet above the micro groove has a spherical shape and $r$ is the radius of the spehere. $P$ is the pitch of the grooves. 


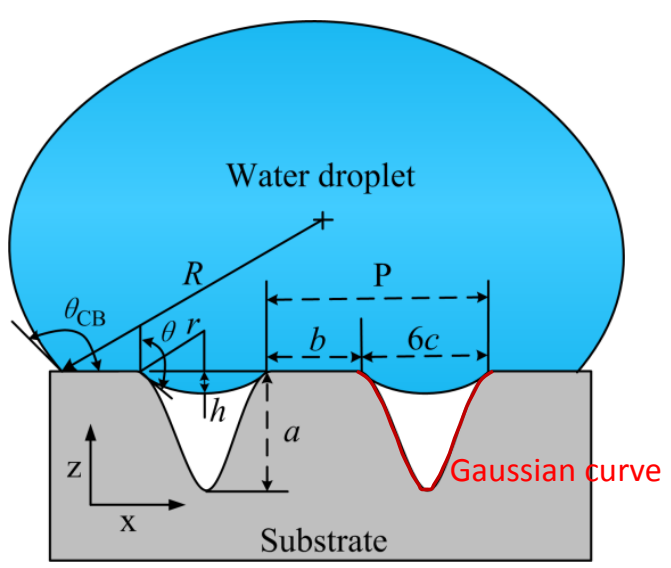

(a) 2D geometric model

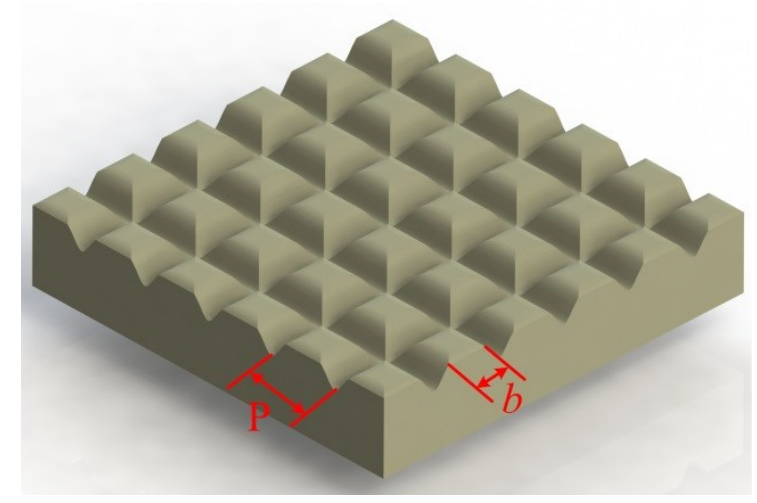

(b) 3D topography

Fig. 1. Geometrical model of laser machined Gaussian grooves at Cassie-Baxter state.

The contact angle at Cassie-Baxter state can be expressed as Eq. (1) [27]:

$$
\cos \theta_{\mathrm{CB}}=-1+f(1+\cos \theta)
$$

where $f$ is the fraction of solid surface area wet by the liquid and it can be described as:

$$
f=\frac{\text { actual contact area }}{\text { planar area }}=\frac{b^{2}}{(b+6 c)^{2}}
$$

From Eq. (1) and (2) it can be seen that the minimum value of $f$ will help the contact angle $\theta_{\mathrm{CB}}$ reaches its maximum value. In order to make the droplet keep a stable Cassie-Baxter state on the micro structured surface, the microstructure should meet some geometrical and chemical constraints, which are listed as follows.

First of all, the sag in height $h$ should be smaller than $a$, thus the droplet cannot contact with the bottom of the micro Gaussian groove. This can be expressed as Eq. (3).

$$
a>h=3 c\left(\frac{1-\sin \theta}{-\cos \theta}\right)
$$

Eq. (3) can be further simplified as: 


$$
3 c\left(\frac{1-\sin \theta}{-\cos \theta}\right)-a \leq 0
$$

Secondly, the state of droplet also depends on the balance between gravity and Laplace pressure (F). The Laplace pressure is the pressure difference between the inside and the outside of a curved surface that forms the boundary between a gas region and a liquid region [29]. The pressure difference is caused by the surface tension of the interface between liquid and gas. Thus, the Laplace pressure should be greater than weight (W) of the droplet. The average droplet weight for every groove and the Laplace pressure (F) are shown in Eq. (5) and (6). The constraint can be expressed by Eq. (7). The pillar can be approximated to possess rectangular shape with side length $b$.

$$
\begin{gathered}
\mathrm{W}=\frac{\rho\left(\frac{4}{3} \pi R^{3} \mathrm{~g}\right)}{\pi R^{2} \sin ^{2} \theta}\left[(b+6 c)^{2}-b^{2}\right] \\
\mathrm{F}=12 \pi \gamma_{\mathrm{lg}} c\left(\frac{1-\sin \theta}{-\cos \theta}\right) \\
\mathrm{W}-\mathrm{F} \leq 0
\end{gathered}
$$

where $\rho$ is water density, $g$ is gravity constant, $R$ is the radius from the centre of the water droplet to the boundary edge of water and Zirconia surface, $\gamma_{\lg }$ is the surface tension between liquid and gas.

Thirdly, principle of the lowest energy is a general rule in nature. Since the mechanical system is trying to find a state of minimum surface free energy [30], the surface free energy of Cassie-Baxter state $\left(\mathrm{E}_{\mathrm{CB}}\right)$ should be smaller than Wenzel state $\left(\mathrm{E}_{\mathrm{W}}\right)$ [28]. The $\mathrm{E}_{\mathrm{CB}}$ and $\mathrm{E}_{\mathrm{W}}$ can be calculated by Eq. (8) and (9). The Eq. (10) is Young's equation [31]. The constraint about the principle of the lowest energy can be expressed by Eq. (11). The side area of micro pillar is processed as a frustum of a pyramid. 


$$
\begin{gathered}
\mathrm{E}_{\mathrm{CB}}=\gamma_{\mathrm{lg}}\left[\frac{18 \pi c^{2}(1-\sin \theta)}{\cos ^{2} \theta}\right]+\gamma_{\mathrm{sl}} b^{2}+4 \gamma_{\mathrm{sg}} \sqrt{a^{2}+9 c^{2}}(b+3 c) \\
\mathrm{E}_{\mathrm{W}}=\gamma_{\mathrm{sl}}\left[b^{2}+4 \sqrt{a^{2}+9 c^{2}}(b+3 c)\right] \\
\gamma_{\mathrm{sg}}=\gamma_{\mathrm{lg}} \cos \theta+\gamma_{\mathrm{sl}} \\
\mathrm{E}_{\mathrm{CB}}-\mathrm{E}_{\mathrm{W}} \leq 0
\end{gathered}
$$

where $\gamma_{\mathrm{sl}}$ is the surface tension between solid and liquid, $\gamma_{\mathrm{sg}}$ is the surface tension between solid and gas.

Finally, there are some additional geometrical constraints. As shown in Fig. 1(b) the relationship of $b, c$ and $\mathrm{P}$ can be expressed as:

$$
b+6 c=\mathrm{P}
$$

For every specimen, the depth of micro groove is also limited by machining depth. Sz can reflect the depth information of the specimens. The depth of the groove should be smaller than $\mathrm{Sz}$ as shown in Eq. (13).

$$
a \leq \mathrm{Sz}
$$

In this optimize problem, Eq. (4), (12) and (13) are linear constraints while Eq. (7) and (11) are nonlinear constraints. MATLAB Optimization Toolbox was used to solve the above optimization problems and the parameters in this study are $92^{\circ}$ intrinsic contact angle of Zirconia $(\theta), 1.06 \mathrm{~mm}$ radius from centre to edge of water and surface $(\mathrm{R})$ and $0.073 \mathrm{~N} / \mathrm{m}$ surface tension between liquid and gas $\left(\gamma_{\mathrm{lg}}\right)$. 
The predicted dimensions of $a, b$ and $c$ for 5 different pitches and its predicted maximum contact angle are shown in Table 1. With the increase of pitch from $60 \mu \mathrm{m}$ (P060) to $180 \mu \mathrm{m}$ (P180), the predicted maximum contact angle decreased from $135^{\circ}$ to $118.3^{\circ}$. In addition, the predicted dimension of $c$ increased from $4.5 \mu \mathrm{m}$ to $7.86 \mu \mathrm{m}$. Thus, the dimension of $b$ also increased from $33 \mu \mathrm{m}$ to $132.8 \mu \mathrm{m}$ in order to make sure the water droplet have a stable Cassie-Baxter state on the microstructures.

Table 1 Optimized dimensions of microstructures for maximizing contact angle

\begin{tabular}{cccccc}
\hline Predicted & $\mathrm{P} 060$ & $\mathrm{P} 090$ & $\mathrm{P} 120$ & $\mathrm{P} 150$ & $\mathrm{P} 180$ \\
items & & & & & \\
\hline$a(\mu \mathrm{m})$ & 87.0 & 101.6 & 76.5 & 133.6 & 76.5 \\
$b(\mu \mathrm{m})$ & 33.0 & 53.7 & 82.4 & 105.1 & 132.8 \\
$6 c(\mu \mathrm{m})$ & 27 & 36.6 & 37.8 & 45 & 47.16 \\
$\theta_{\mathrm{CB}}\left(^{\circ}\right)$ & 135 & 131 & 123 & 121 & 118.3 \\
\hline
\end{tabular}

\section{Microstructure design and experimental details}

The microstructured surface on zirconia was generated by laser ablation in order to verify the predicted structure dimension and predicted maximum contact angle.

\subsection{Pattern design and sample preparation}

Five zirconia specimens were machined in this experiment. Each specimen has a diameter of $10 \mathrm{~mm}$ and thickness of $1 \mathrm{~mm}$. Before laser machining, the specimens were rinsed with ethanol in ultrasonic cleaning equipment for 10 minutes. Micro pillar-array designed for this experiment is shown in Fig. 1 (b). The pitch for different specimens varies from $60 \mu \mathrm{m}$ to 180 $\mu \mathrm{m}$ for the five specimens. For each specimen, the pitch $(P)$ is the same in the vertical and horizontal directions. 


\subsection{Experimental setup}

The laser machining experiments were carried out on a hybrid ultra-precision machine equipped with a nanosecond pulsed Ytterbium fibre laser (IPG YLP-V2-100). The experimental setup is shown in Fig. 2. The laser head is mounted on the $\mathrm{Z}$ axis and its laser wavelength is $1064 \mathrm{~nm}$. The laser source has nominal average output power of $20 \mathrm{~W}$ and maximum pulse repetition rate of $200 \mathrm{kHz}$. During operation the laser beam passes through a lens and focuses onto the surface of specimen and the workpiece is mounted on precision XY-C axes stages.

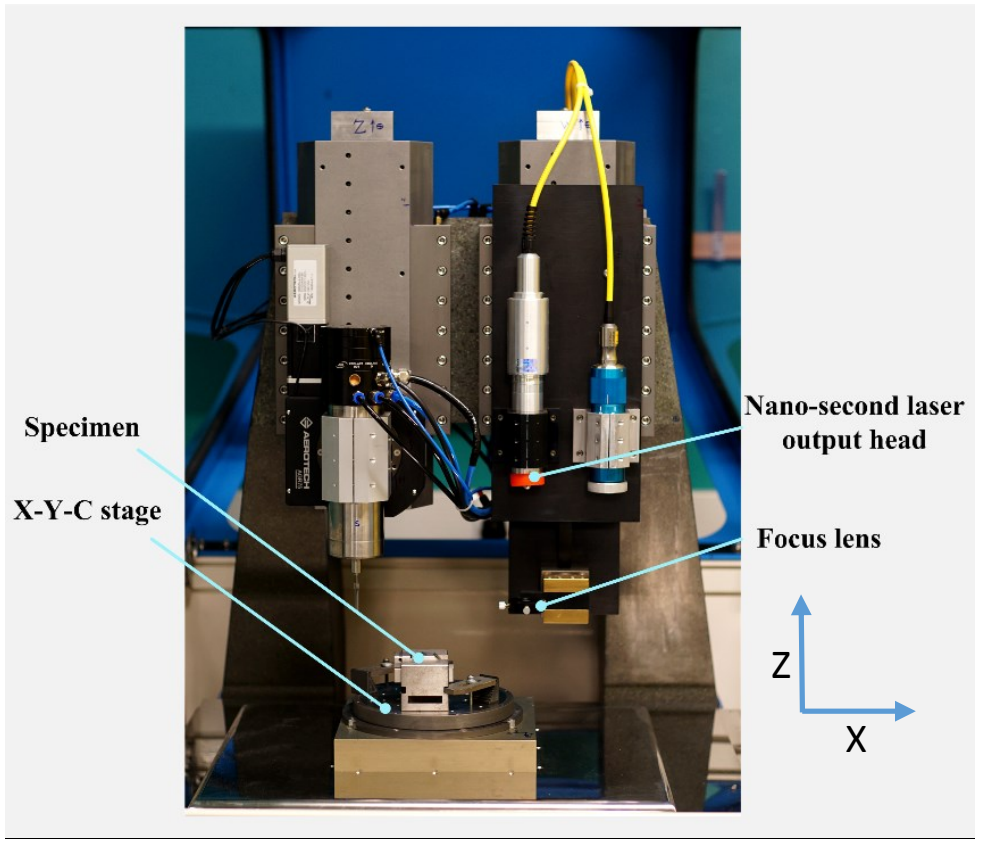

Fig. 2. Experimental setup.

First of all, unidirectional laser machining was employed to generate Gaussian grooves on the sample surface by horizontal machining strategy. After finishing the whole microgrooves, vertical laser machining with same step distance was then employed to obtain the final pillar array. The laser machining parameters are laser power of $8 \mathrm{~W}$, pulse repetition rate of $100 \mathrm{~K}$ with federate of $200 \mathrm{~mm} / \mathrm{min}$ for the $\mathrm{X}$ and $\mathrm{Y}$ linear stages. 
After laser machining, the specimens were rinsed with deionized water in ultrasonic cleaning equipment for half an hour to remove the molten slag on the surface. Then, these specimens were degreased in a 30-min ultrasonic bath in acetone and ethanol respectively. Finally, these specimens were dried in an oven. Before measuring the contact angle, these specimens were silanized in a vacuum oven using silane reagent $(1 \mathrm{H}, 1 \mathrm{H}, 2 \mathrm{H}, 2 \mathrm{H}-$ Perfluorooctyltriethoxysilane, $97 \%$, Alfa Aesar Ltd), at $100^{\circ} \mathrm{C}$ for 12 hours to reduce their surface free energies.

\section{Results}

\subsection{Analysis of surface morphology}

The morphology and surface roughness of the laser structured surface were measured by an optical 3D surface measurement microscope (Alicona G4) and a scanning electron microscope (SEM) (Hitachi TM-1000). The images on the left in Fig. 3 are the 3D surface morphologies with measurement field of view of $710 \mu \mathrm{m} \times 540 \mu \mathrm{m}$. Heights of each measured microstructure are indicated in the colour scale bar. The recast layers of the melted work materials were observed to form on the micro pillars. However, the micro pillar became more and more clear and the micro grooves were gradually well separated with the increase of pitch. SEM images of the machined surfaces (all measured under 500× Magnifications with scale bars of $50 \mu \mathrm{m}$ ) are shown on the right in Fig.3. Thermally induced cracks were observed on these surfaces and became more apparent with the increase of laser power.
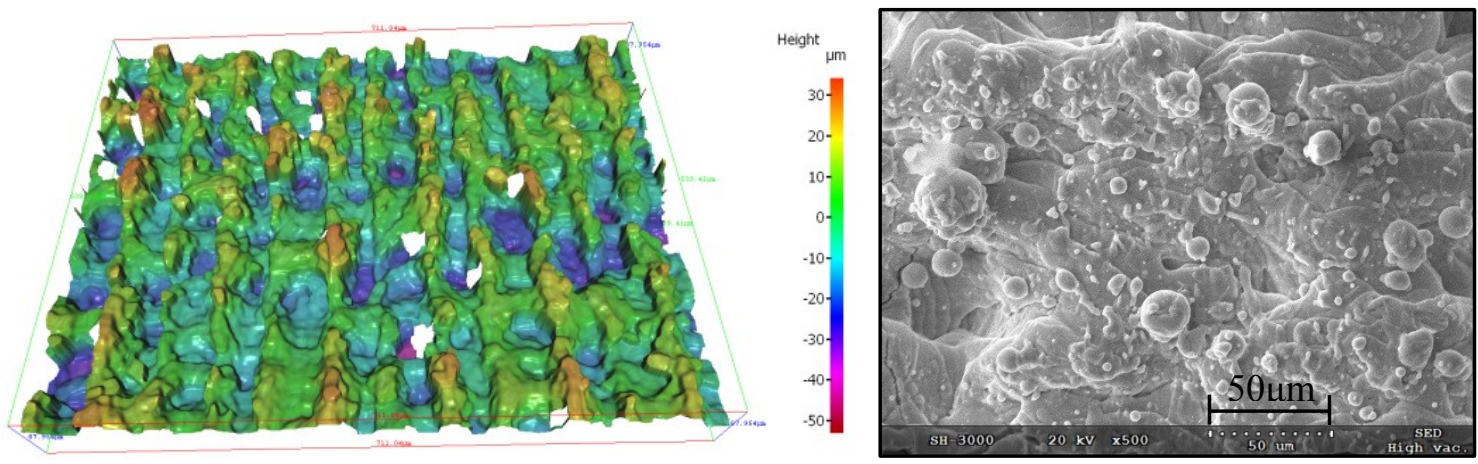
(a) $\mathrm{P} 060$
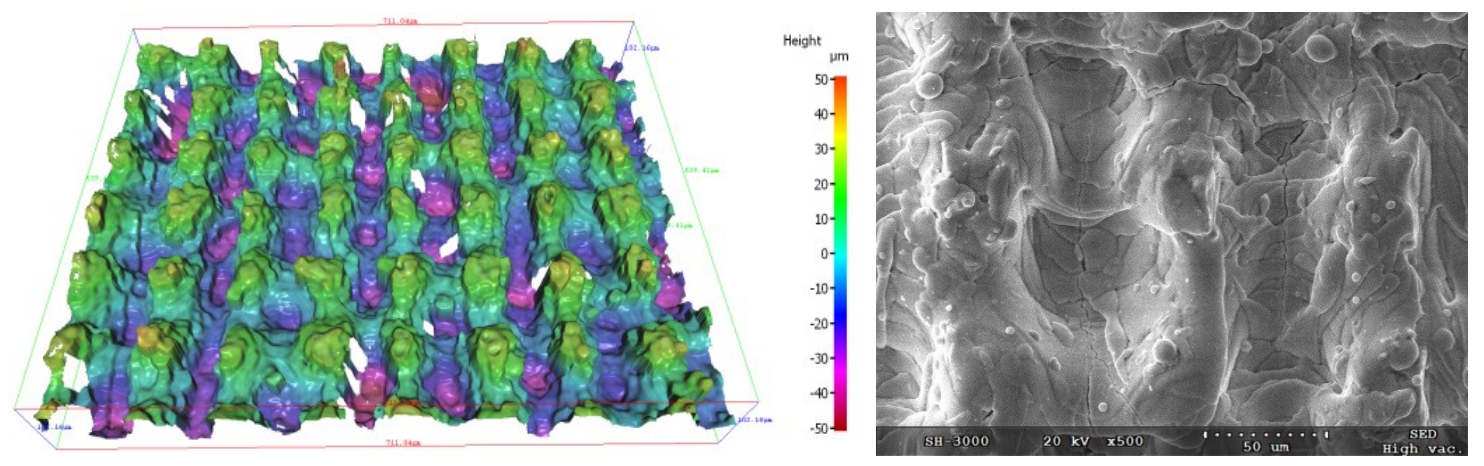

(b) P090
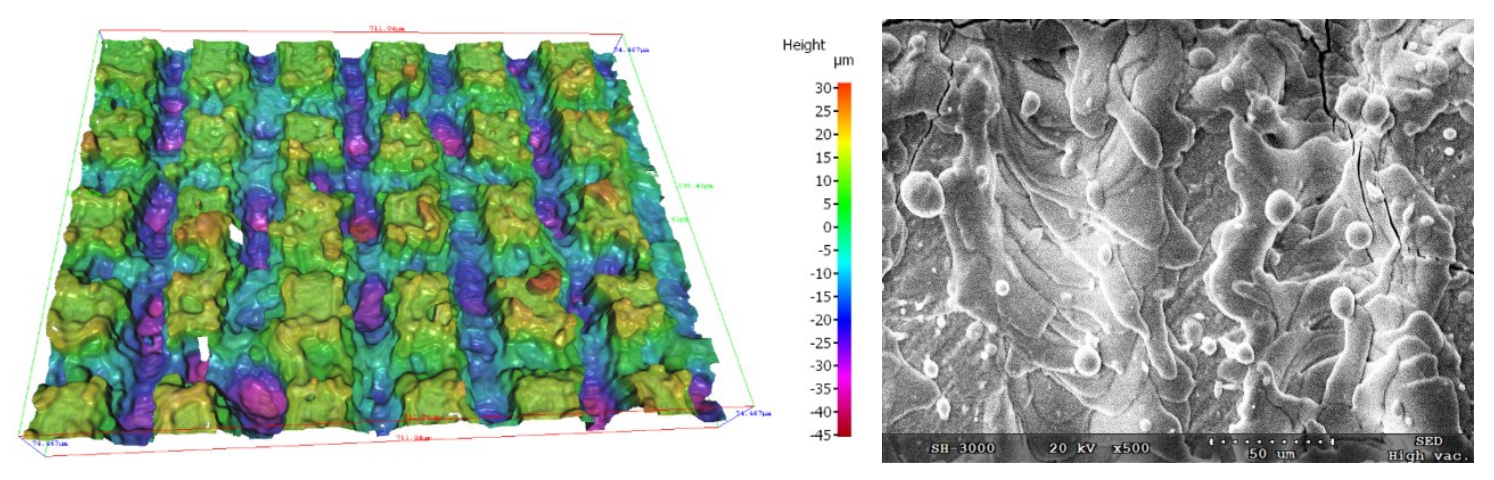

(c) P120
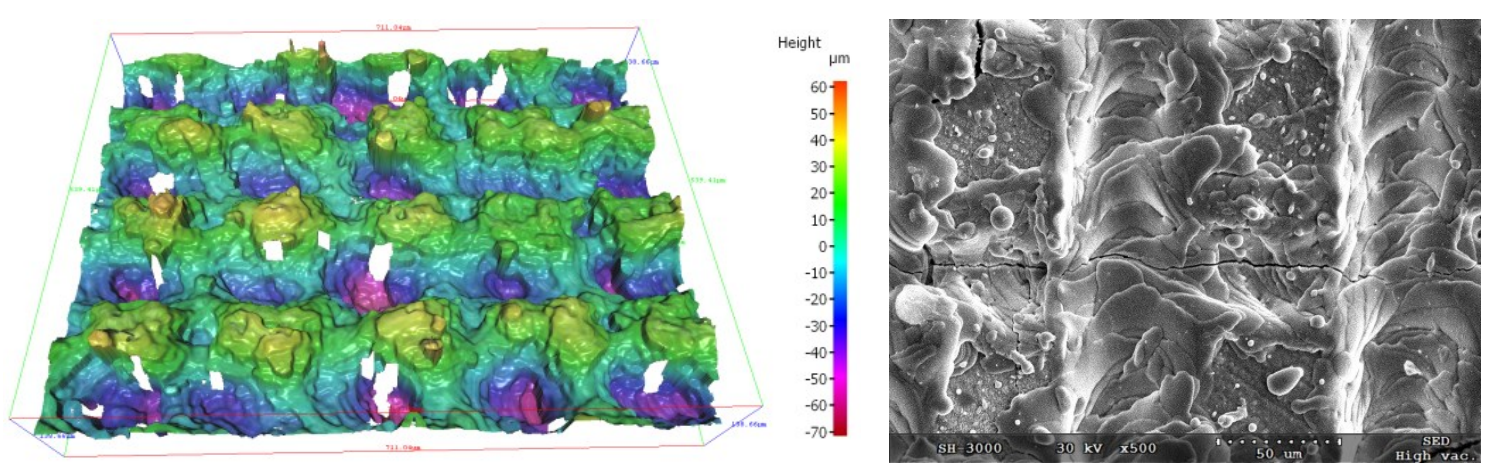

(d) P150 

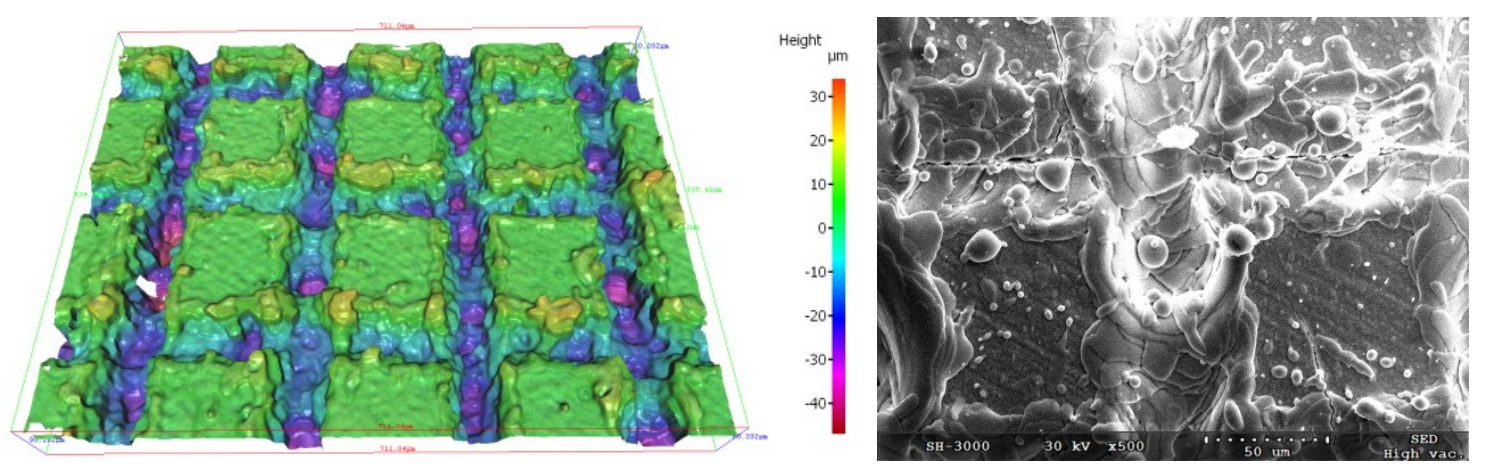

(e) P180

Fig. 3. Surface morphologies of specimens with different pitches.

\subsection{Analysis of surface hydrophobicity}

In this work, apparent contact angle on surfaces was measured by a drop shape analyser (Kruss Ltd). A $5 \mu \mathrm{L}$ droplet of deionised water was dropped on the smooth milled specimen at atmospheric conditions. The side view was captured by an industrial camera and the contact angle can be determined by image processing software. As shown in Fig. 4 (a), the contact angle $\theta$ is $92^{\circ}$ for the smooth specimen. For each specimen, the contact angle of the water droplet was measured three times and the average value was adopted.

Fig.4 (b) to (f) shows the captured image of the droplets in the contact angle measurements. In the results, the increased pitch leads to a decrease of contact angle from $133.1^{\circ}$ to $115.3^{\circ}$. However, an exception was observed for the specimen P090 which has a contact angle of 104.5 $5^{\circ}$ The mechanism of this abnormal phenomenon will be explored in the next section. 


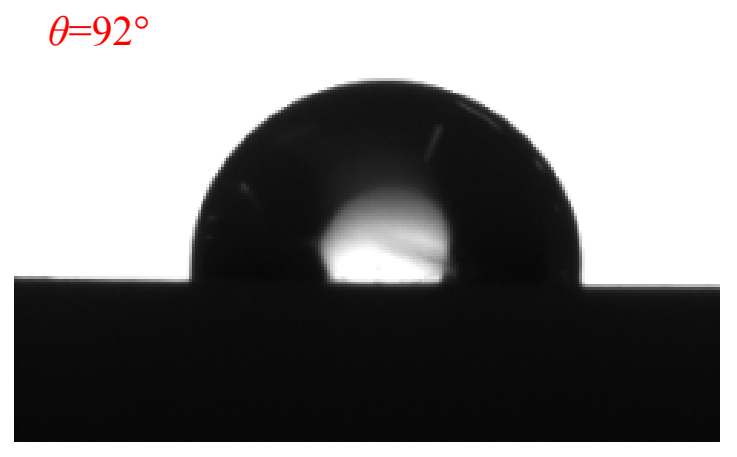

(a) Original surface

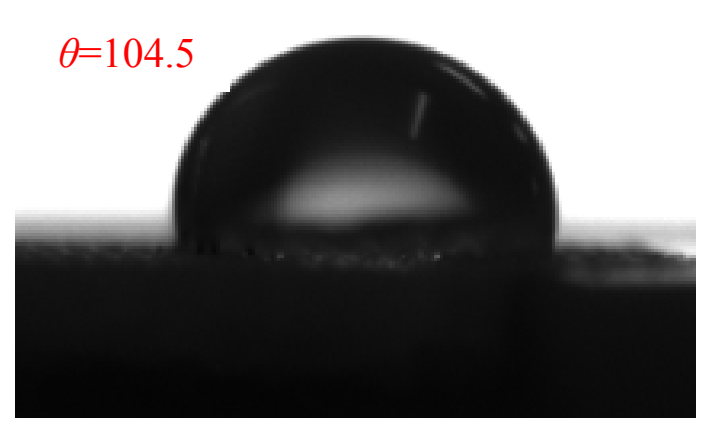

(c) P090

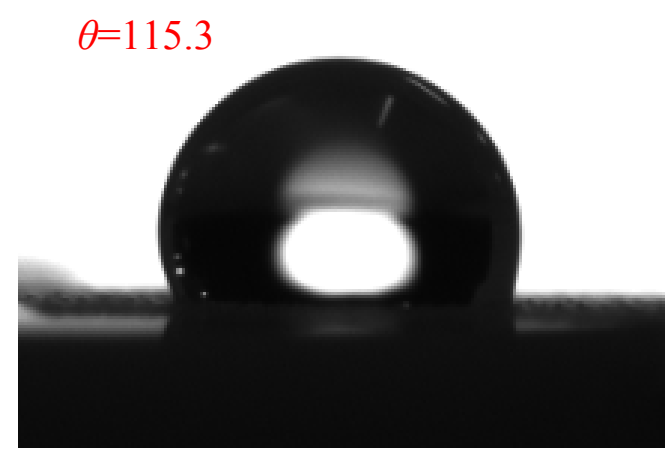

(e) P150

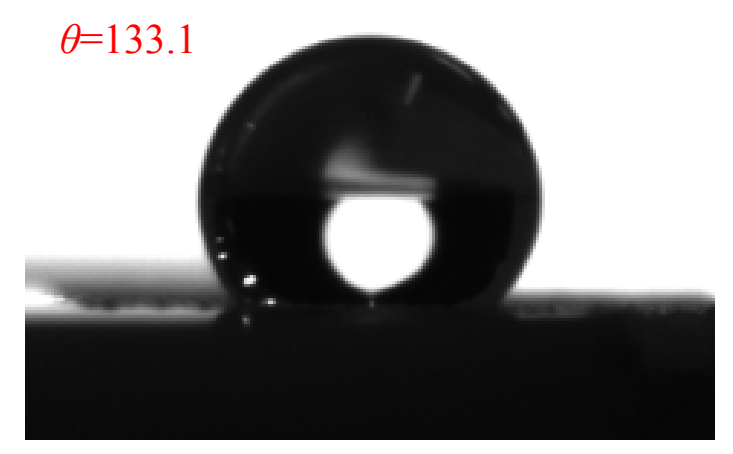

(b) P060

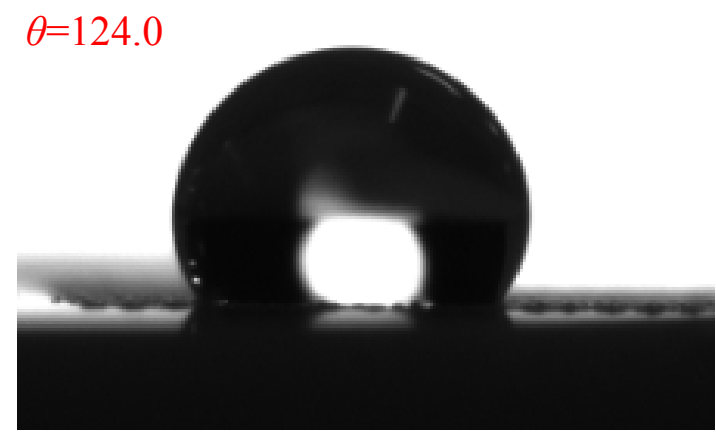

(d) P120

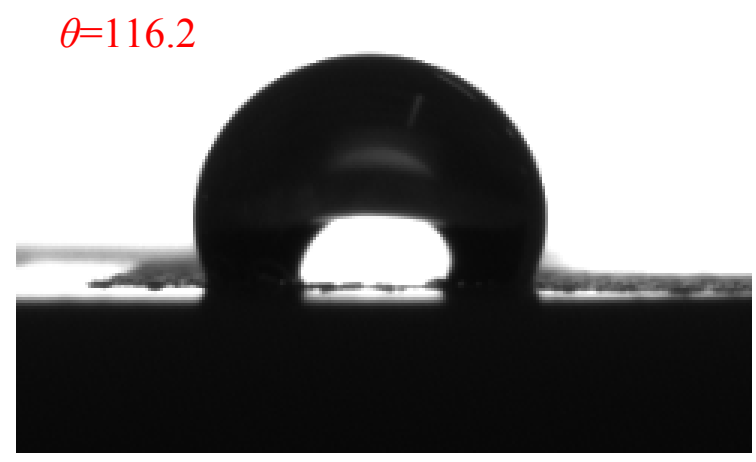

(f) P180

Fig. 4. Water droplet shapes for different specimens.

Fig. 5 shows the comparison result between the predicted maximum contact angle and experimental value. First of all, the predicted and the measured contact angle both show trend of decrease with the increase of pitch of the micro pillars. For specimens, P060, P120, P150 and P180, the difference between the predicted maximum contact angle and the measured 
values are less than 5\%. However, for specimen P090, the actual value of contact angle is far from the predicted maximum value, which has $20.2 \%$ difference from the the predicted maximum contact angle $\left(131^{\circ}\right)$. P060 specimen, with a contact angle of $133.1^{\circ}$, has the best hydrophobicity among all specimens in the experiment. The fitting curve of the predicted maximum contact angle indicates that microstructure with pitch smaller than $60 \mu \mathrm{m}$ will result in even higher contact angle of above $133.1^{\circ}$. This will be verified by additional machining experiment to be described in the follow section.

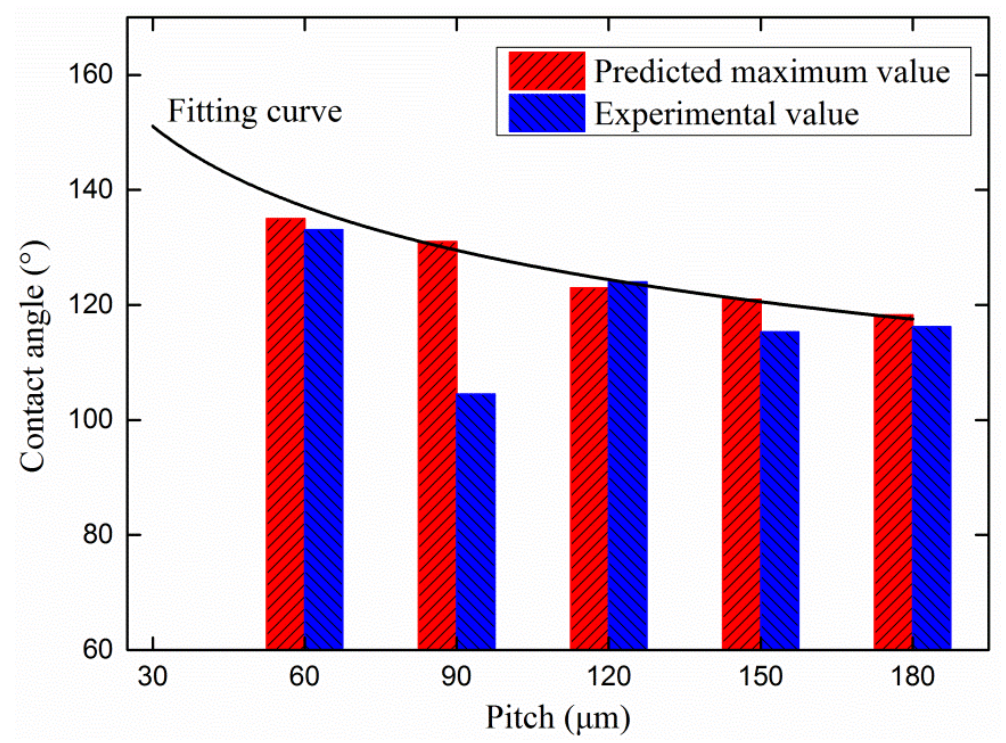

Fig. 5. Comparison between the predicted maximum contact angle and measured value.

\section{Discussions}

The result in Fig. 5 shows that most of specimens have little deviations to the predicted maximum contact angle. It also shows that the contact angle increases when the pitch of the microstructures decrease. In this section, the mechanism and reason of these two findings will be discussed. Additional experimental results will also be presented in order to further evaluate the prediction model.

Since the contact angle is affected by microstructure pitch, the 2D surface profiles extracted and measured under different pitches. The results are shown in Fig. 6 explaining some 
contact angle of specimens are close to the predicted values and the others are far away from the predicted maximum value. According to Eq. (12), the sum of $b$ and $6 c$ are constant value, which is equal to the pitch P. In Fig. 6, the width of pillar $b$ was measured and compared with the predicted value. For every specimen, line $Z=0$ is the center line of least squares interface. Therefore, the average width of pillar $(b)$ at position $\mathrm{Z}=0$ is used to represent the width of pillar.

The cross-sectional profiles were taken in the middle of the microstructure along the latitude direction. For P060, the average $b$ is $31.03 \mu \mathrm{m}$, which has $5.9 \%$ difference from the predicted value of $b(33 \mu \mathrm{m})$. For P120, the average $b$ is $82.5 \mu \mathrm{m}$, which is almost same as the predicted value of $b(82.4 \mu \mathrm{m})$. In addition, the specimens P150 and P180 also have small deviations to the predicted value of $b$, which are $1.6 \%$ and $1.1 \%$, respectively. Thus, the dimensions of actual microstructures are close to the predicted dimensions and that is the major reason of experimental contact angle close to the predicted maximum value. However, for P090, the average $b$ is $40.53 \mu \mathrm{m}$ which has $24.5 \%$ difference from the predicted value of $b(53.7 \mu \mathrm{m})$. The large deviation to the designed dimensions of microstructure is the reason of causing such big differences between the measured and predicted contact angles.

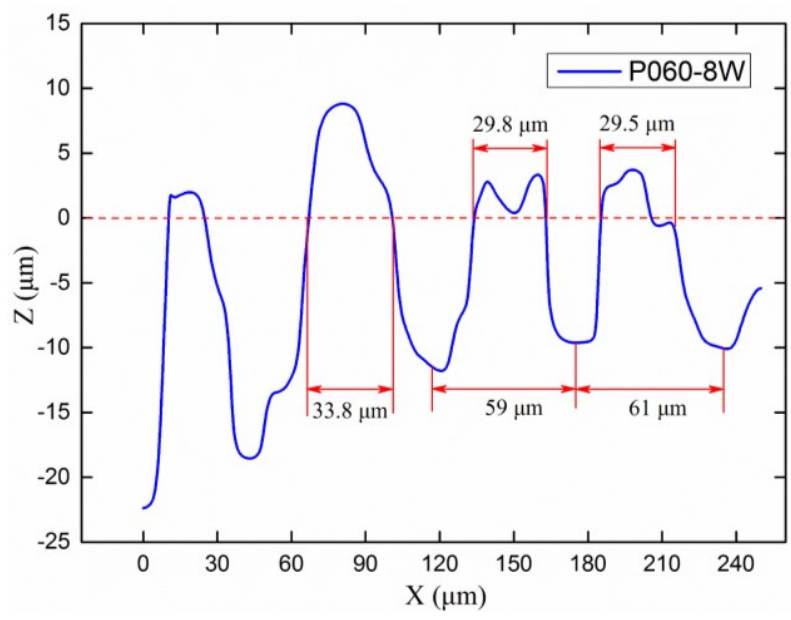


(a) P060

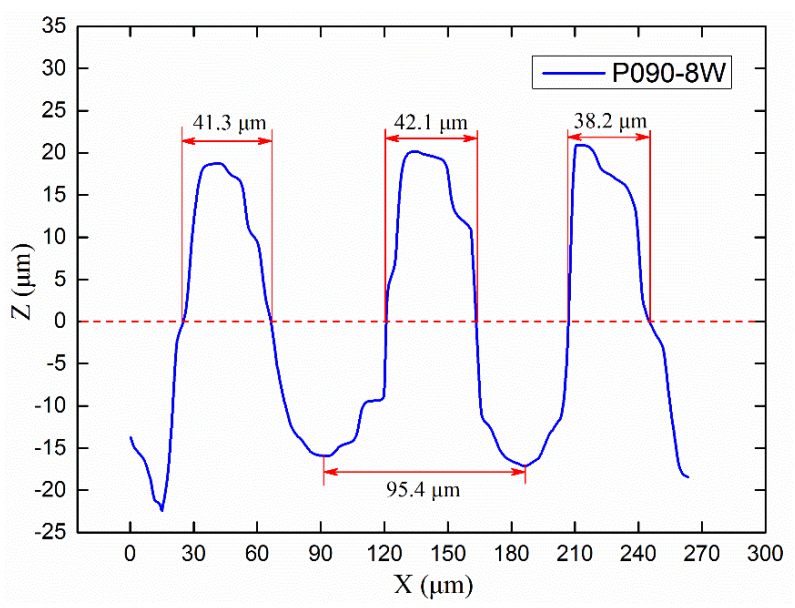

(b) P090

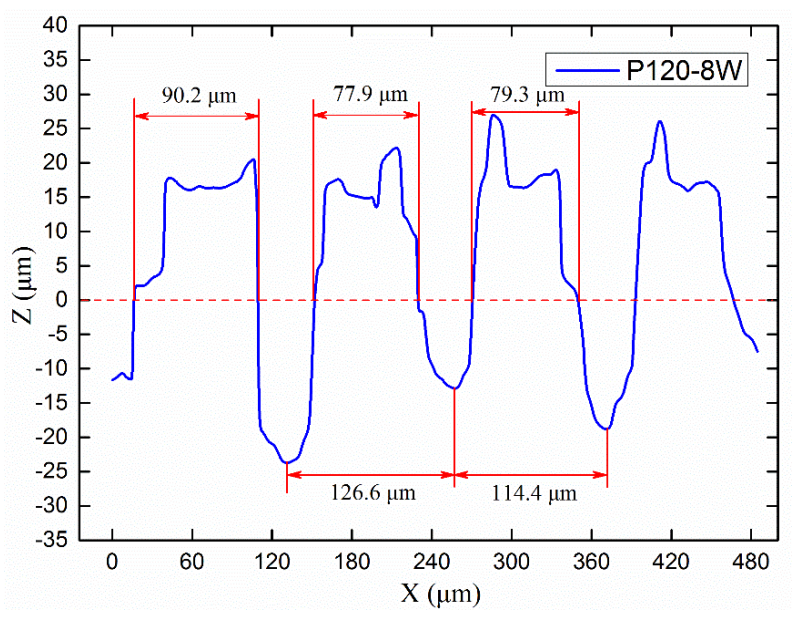

(c) P120

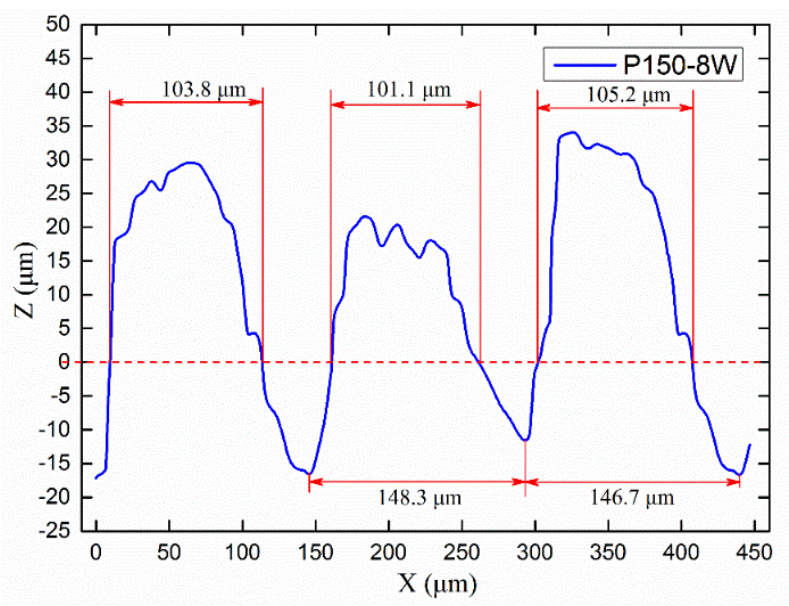


(d) P150

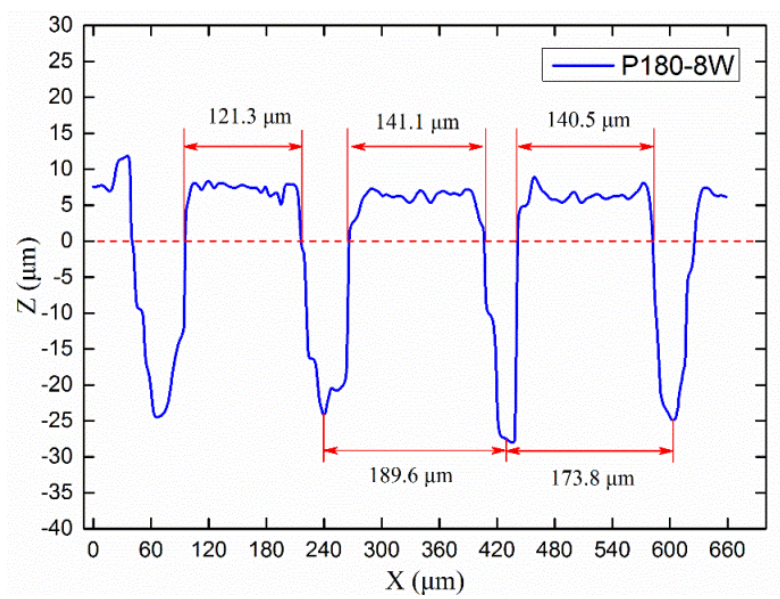

(e) P180

Fig. 6. Surface profiles of nanosecond laser machined specimens.

In order to verify the finding on the contact angle increasing with the decrease of pitch of the microstructures, additional experiments were conducted, in which a specimen with a smaller pitch of $30 \mu \mathrm{m}(\mathrm{P} 030)$ at a laser power of $8 \mathrm{~W}$ was machined. As the minimum spot size of this laser is about $15 \mu \mathrm{m}$ the minimum pitch of the machine microstructure will be $30 \mu \mathrm{m}$. The SEM image of specimen P030 is shown in Fig.7. Very tiny cracks could still be observed. The measured contact angle of $\mathrm{P} 030$ is about $155.7^{\circ}$ as shown in Fig. 8 . It is, therefore the best hydrophobicity among all specimens and can be classified as a superhydrophobic surface. Thus, it proves that microstrucutres with smaller pitch will have better superhydrophobicity than large pitch. 


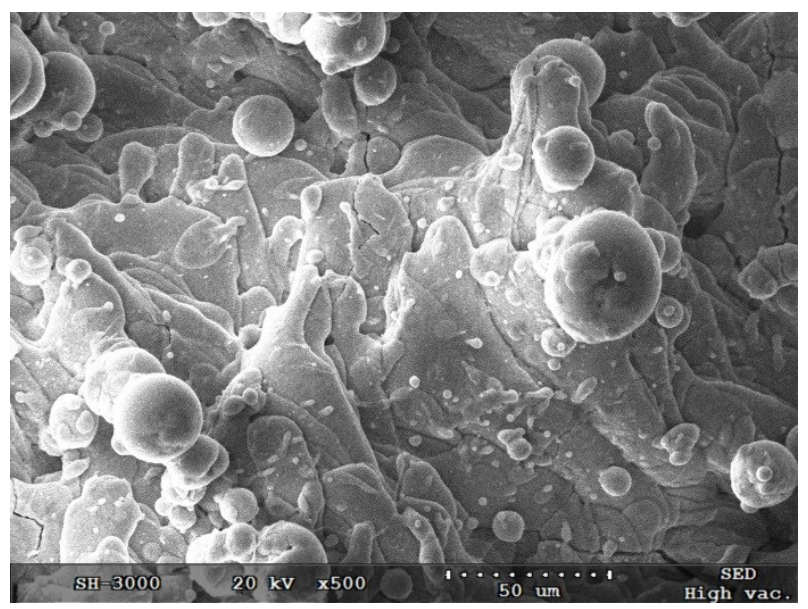

Fig. 7 SEM image of P030

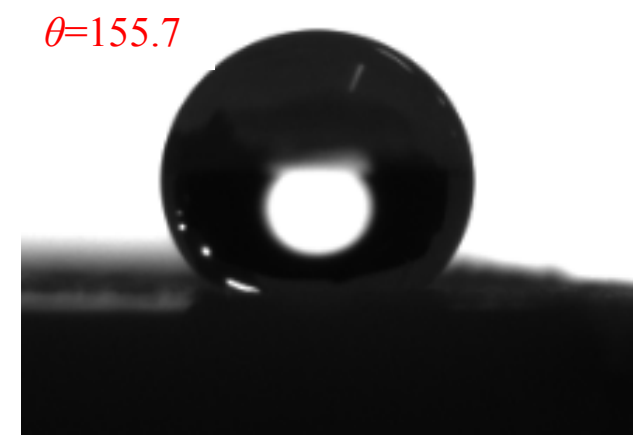

Fig. 8 Water droplet shapes for P030

In previous laser machining experiment, the major dimensional deviation for the specimen P090 is the width of micro pillar $b$ which is smaller than the predicted value. Lower laser power is expected to yield lower material removal rates. Thus, the reduction of laser power is an effective method to increase micro pillar width. In additional experiments, a lower laser power of $4 \mathrm{~W}$ was used to manufacture a new specimen with pitch of $90 \mu \mathrm{m}$ (P090-4W). The measured surface profile of P090-4W taken in the middle of the microstructure along the latitude direction is shown in Fig. 9. The average $b$ is $57.1 \mu \mathrm{m}$, which is only $6.3 \%$ difference from the predicted value of $b(53.7 \mu \mathrm{m})$. After post processing, its experimental contact angle 
is $127.4^{\circ}$ as shown in Fig. 9 (b). It is now very close (only $2.7 \%$ difference) to the predicted maximum contact angle $\left(131^{\circ}\right)$.

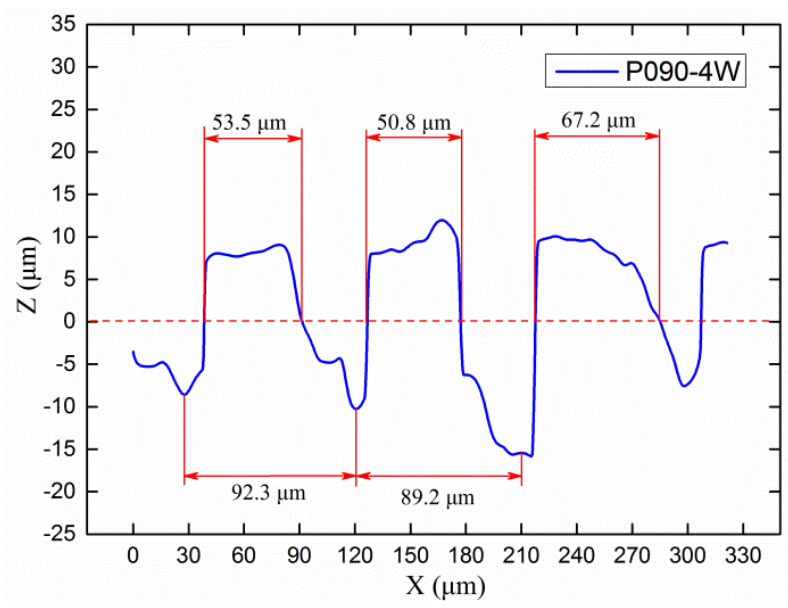

(a)Surface profiles of P090-4W

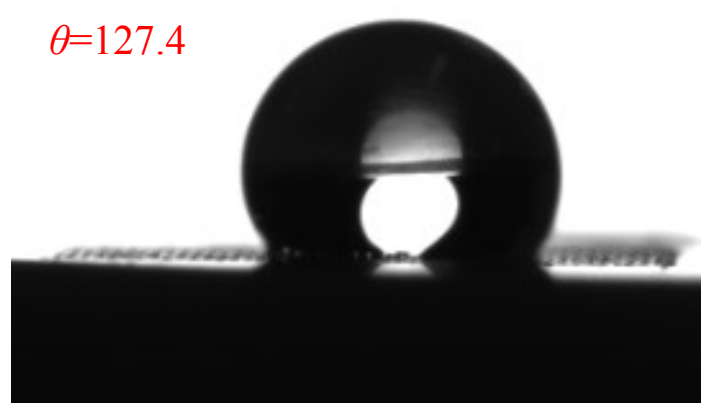

(b) Water droplet shapes

Fig. 9. Surface profiles and water droplet shapes for P090-4W

Fig. 10 shows a complete comparison between experimental and predicted maximum contact angles in this study. For all specimens, the experimental contact angle has a small deviation (less than 5\%) from the predicted maximum value. This indicates that the proposed contact angle prediction model can be used to obtain best dimensions of microstructure and predict its maximum contact angle for superhydrophobicity. 


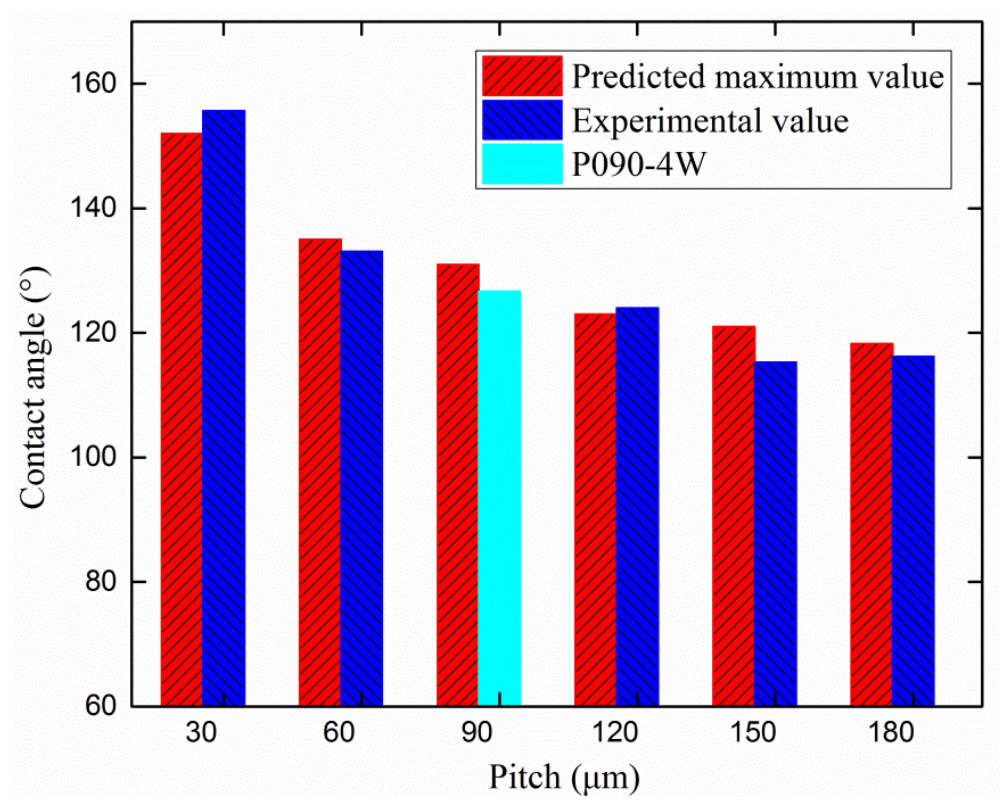

Fig.10 The comparison between experimental and predicted maximum contact angle.

\section{Conclusions}

In this work, the prediction model of contact angle for laser machined microstructured Zirconia surfaces was established. Together with the theoretical constraints for stable CassieBaxter state, the prediction model can be used to optimize the dimensions of microstructures for maximising surface hydrophobicity. The experimental results show that the proposed model can accurately predict the contact angle within 5\% deviation. Both experimental and theoretical prediction results show that the contact angle will increase with the decrease of the pitch of microstructures. Superhydrophobicity was successfully obtained with the maximum contact angle of $155.7^{\circ}$ on a micro structured surface $(\mathrm{P} 030-8 \mathrm{~W})$ on Zirconia with a pitch of $30 \mu \mathrm{m}$ machined under a laser power of $8 \mathrm{~W}$. In the future, the effects of other geometrical parameters such as depth and diameter of micro-dimple will be investigated in order to find the best microstructures for superhydrophobicity. The measures to eliminate/minimize microcracks in laser processing need to be investigated as well. 


\section{Acknowledgements}

This research was undertaken in the context of MICROMAN project ("Process Fingerprint for Zero-defect Net-shape MICROMANufacturing”, http://www.microman.mek.dtu.dk/). MICROMAN is a European Training Network supported by Horizon 2020, the EU Framework Programme for Research and Innovation (Project ID: 674801). The authors would also gratefully acknowledge the financial support from the EPSRC (EP/K018345/1) for this research

\section{Reference}

[1] Cavalcanti AN, Foxton RM, Watson TF, Oliveira MT, Giannini M, Marchi GM. Bond strength of resin cements to a zirconia ceramic with different surface treatments. Oper Dent 2009; 34: 280-287

[2] Yilmaz H, Aydin C, Gul BE. Flexural strength and fracture toughness of dental core ceramics. J Prosthet Dent 2007; 98: 120-128

[3] Scotti R, Kantorski KZ, Monaco C, Valandro LF, Ciocca L,Bottino MA. SEM evaluation of in situ early bacterial coloni-zation on a Y-TZP ceramic: a pilot study. Int J Prosthodont 2007;20:419-422.

[4] M. Hisbergues, S. Vendeville, P. Vendeville, Review zirconia: established facts and perspectives for a biomaterial in dental implantology, J Biomed Mater Res: Appl Biomater, 88B (2009), pp. 519-529

[5] A. Pier-Francesco, R. Adams, M. Waters, D. Williams, Titanium surface modification and its effect on the adherence of Porphyromonas gingivalis: an in vitro study, Clinical Oral Implants Research, 17 (2006), pp. 633-637 
[6] R. Blossey, Self-cleaning surfaces — virtual realities, Nat. Mater. 2 (2003) 301-306, http://dx.doi.org/10.1038/nmat856

[7] B. Wang, Y. Zhang, L. Shi, J. Li, Z. Guo, Advances in the theory of superhydrophobic surfaces, J. Mater. Chem. 22 (2012) 20112. doi:10.1039/c2jm32780e.

[8] K. Liu, X. Yao, L. Jiang, Recent developments in bio-inspired special wettability., Chem. Soc. Rev. 39 (2010) 3240-55. doi:10.1039/b917112f.

[9] B.S. Yilbas, H. Ali, M. Khaled, N. Al-Aqeeli, N. Abu-Dheir and K.K. Varanasi, Characteristics of laser textured silicon surface and effect of mud adhesion on hydrophobicity, Applied Surface Science 351 (2015) 880-888

[10] S. Zhenyu, L. Zhanqiang, S. Hao, Z. Xianzhi, Prediction of contact angle for hydrophobic surface fabricated with micro-machining based on minimum Gibbs free energy, Appl. Surf. Sci. 364 (2016) 597-603. doi:10.1016/j.apsusc.2015.12.199.

[11] Y. Cai, Hydrophobicity of pyramid structures fabricated by micro milling. 2017 World Congr. Micro Nano Manuf. (2017).

[12] N. J. Shirtcliffe, G. McHale, M.I. Newton, G. Chabrol, C.C. Perry, Dual-scale roughness produces unusually water-repellent surfaces, Adv. Mater. 16 (2004) 1929-1932. doi:10.1002/adma.200400315.

[13] K. K. S. Lau, J. Bico, K. B. K. Teo, M. Chhowalla, G. A. J. Amaratunga, W.I. Milne, G.H. McKinley, K.K. Gleason, Superhydrophobic Carbon Nanotube Forests, Nano Lett. 3 (2003) 1701-1705. doi:10.1021/n1034704t

[14] R. Fürstner, W. Barthlott, C. Neinhuis, P. Walzel, Wetting and self-cleaning properties of artificial superhydrophobic surfaces, Langmuir. 21 (2005) 956-961. doi:10.1021/la0401011. 
[15] D.M. Chun, C.V. Ngo, K.M. Lee, Fast fabrication of superhydrophobic metallic surface using nanosecond laser texturing and low-temperature annealing, CIRP Ann. - Manuf. Technol. 65 (2016) 519-522. doi:10.1016/j.cirp.2016.04.019.

[16] D. V Ta, A. Dunn, T.J. Wasley, R.W. Kay, J. Stringer, P.J. Smith, C. Connaughton, J.D. Shephard, Nanosecond laser textured superhydrophobic metallic surfaces and their chemical sensing applications, Applied Surface Science, 357 (2015) 248-254.

doi:10.1016/j.apsusc.2016.04.056

[17] M. Martínez-Calderon, A. Rodríguez, A. Dias-Ponte, M.C. Morant-Miñana, M. GómezAranzadi, S.M. Olaizola, Femtosecond laser fabrication of highly hydrophobic stainless steel surface with hierarchical structures fabricated by combining ordered microstructures and LIPSS, Appl. Surf. Sci. 374 (2016) 81-89. doi:10.1016/j.apsusc.2015.09.261.

[18] B. Zheng, G. Jiang, W. Wang, X. Mei, Fabrication of superhydrophilic or superhydrophobic self-cleaning metal surfaces using picosecond laser pulses and chemical fluorination., Radiat. Eff. Defects Solids. 171 (2016) 461-473.

doi:10.1080/10420150.2016.1211658.

[19] B. Wu, M. Zhou, J. Li, X. Ye, G. Li, L. Cai, Superhydrophobic surfaces fabricated by microstructuring of stainless steel using a femtosecond laser, Appl. Surf. Sci. 256 (2009) 6166. doi:10.1016/j.apsusc.2009.07.061.

[20] R. Jagdheesh, J. J. García-Ballesteros, J. L. Ocaña, One-step fabrication of near superhydrophobic aluminum surface by nanosecond laser ablation, Appl. Surf. Sci. 374 (2016) 2-11. doi:10.1016/j.apsusc.2015.06.104.

[21] F.H. Rajab, P. Benson, L. Li, K.A. Whitehead, Picosecond Laser Surface Micro / Nano Texturing of Stainless Steel as a Method to Reduce the Adhesion of Bacteria, Proceedings of LPM2017 - the 18th International Symposium on Laser Precision Microfabrication 
[22] P. Tang, W. Zhang, Y. Wang, B. Zhang, H. Wang, C. Lin and L. Zhang, Effect of superhydrophobic surface of titanium on Staphyococcus aureus adhesion, J.Nanomater., 2011, article ID 178921

[23] M.H. Kwon, H.S. Shin, C.N. Chu, Fabrication of a super-hydrophobic surface on metal using laser ablation and electrodeposition, Appl. Surf. Sci. 288 (2014) 222-228. doi:10.1016/j.apsusc.2013.10.011.

[24] Z. Yang, Y.L. Tian, C.J. Yang, F.J. Wang, X.P. Liu, Modification of wetting property of Inconel 718 surface by nanosecond laser texturing, Appl. Surf. Sci. 414 (2017) 313-324. doi:10.1016/j.apsusc.2017.04.050.

[25] B.S. Yilbas, Laser treatment of zirconia surface for improved surface hydrophobicity, Journal of Alloys and Compounds 625 (2015) 208-215

[26] Bekir Sami Yilbas, Laser texturing of zirconia surface with presence of TiC and B4C: Surface hydrophobicity, metallurgical, and mechanical characteristics, Ceramics International 40 (2014) 16159-16167

[27] B.D. Cassie, Wettability of porous surfaces, (1944) 546-551. doi:10.1039/tf9444000546.

[28] R.N. Wenzel, Resistance of Solid Surfaces To Wetting By Water, Ind. Eng. Chem. 28 (1936) 988-994. doi:10.1021/ie50320a024.

[29] K.M. Graf K, ed., Physics and chemistry of interfaces, John Wiley \& Sons, 2006.

[30] https://en.wikipedia.org/wiki/Surface_tension\#cite_note-s_z-7.

[31] T. Young, An Essay on the Cohesion of Fluids, Philos. Trans. R. Soc. London. 95 (1805) 65-87. doi:10.1098/rstl.1805.0005. 